\title{
Optimal Competitive Algorithms for Opportunistic Spectrum Access
}

\author{
Nicholas B. Chang and Mingyan Liu
}

\begin{abstract}
We consider opportunistic spectrum access (OSA) strategies for a transmitter in a multichannel wireless system, where a channel may or may not be available and the transmitter must sense/probe the channel to find out before transmission. Applications for this work include joint probing and transmission for a secondary user in a cognitive radio network. Limited by resources, e.g., energy and time, the transmitter must decide on a subset of a potentially very large number of channels to probe and can only use for transmission those that have been found to be available. In contrast to previous works, we do not assume the user has a priori knowledge regarding the statistics of channel states. The main goal of this work is to design robust strategies that decide, based only on knowledge of the channel bandwidths/data rates, which channels to probe. We derive optimal strategies that maximize the total expected bandwidth/data rate in the worst-case, via a performance measure in the form of a competitive regret (ratio) between the average performance of a strategy and a genie (or omniscient observer). This formulation can also be viewed as a two-player zero-sum game between the user and an adversary which chooses the channel state that minimizes the user's gain. We show that our results correspond to a Nash equilibrium (in the form of a mixed strategy) in this game. We examine the performance of the optimal strategies under a wide range of system parameters and practical channel models via numerical studies.
\end{abstract}

Index Terms-software-defined radio, cognitive radio, channel probing, worst-case performance, competitive analysis, twoplayer zero-sum game, Nash equilibrium, online algorithms, randomized algorithms, mixed strategy

\section{INTRODUCTION}

In this paper, we examine optimal channel sensing/probing strategies for opportunistic spectrum access (OSA), where a transmitter seeks to maximize its achievable data rate by opportunistically transmitting over a select subset of a potentially large number of channels. This is done by optimally constructing a channel sensing/probing strategy to find out which channels are available before transmitting. This problem is motivated by wireless systems where a transmitter is supplied with more channels than needed for transmission.

This work was completed while N.Chang was with the University of Michigan. He is now with the Advanced Sensor Techniques Group at MIT Lincoln Laboratory, 244 Wood Street, Lexington, MA 02420-9108. Email: nchang@11.mit.edu.

M. Liu is with the Department of Electrical Engineering and Computer Science, University of Michigan, Ann Arbor, MI 48109-2122. Email: mingyan@eecs.umich.edu.

This work is supported by NSF Award ANI-0238035, ONR grant N0001403-1-0232, and through collaborative participation in the Communications and Networks Consortium sponsored by the U.S. Army Research Laboratory under the Collaborative Technology Alliance Program. Cooperative Agreement DAAD19-01-2-0011.
For example, software defined radio [1] and cognitive radio systems [2] may provide users with multiple channels (e.g. tunable frequency bands and modulation techniques) by means of a programmable hardware which is controlled by software. Channel quality is in general time-varying due to factors such as fading, interference, and the presence of other users (e.g. primary users in a cognitive radio network). Therefore the ability to probe or sense channel quality before transmission can help transmitters select the best one(s) to use. One method of channel probing is through the exchange of control packets between transmitter and receiver [3]. Another method is to use a spectrum sensor [2] at the physical layer.

Due to constraints on time, energy, and other resources, a transmitter may only be able to probe a limited number of channels. Therefore, it is imperative that it judiciously select the right channels to probe. If the transmitter has $a$ priori knowledge on the probability distribution describing the states of these channels, then it can design strategies which maximize the expected transmission gain. Recent works such as [4], [5], [6] have studied the tradeoff between probing to gain more information about channel quality and maximizing transmission gain. On the other hand, if the transmitter cannot accurately estimate the distribution of channel states, e.g., due to insufficient information or because the underlying distribution is changing rapidly over time, then it may be unreasonable to assume such a priori knowledge. It is therefore necessary in such cases to design channel probing and transmission strategies which are more robust and do not require a priori knowledge of the channel statistics.

Motivated by these practical considerations, in this paper we use competitive analysis [7] methods to seek strategies that perform well in the worst case (to be defined more precisely in Section II) for the following problem. A transmitter has to decide which subset (up to $K$ channels) of $N$ channels to probe, whereby it can only transmit using those channels that have been probed to be available, and its performance is measured against a genie who knows exactly which channels are available. To the best of our knowledge, this is the first work which analyzes opportunistic spectrum access policies using a competitive framework. This formulation can also be viewed as a two-player strictly competitive (or zero-sum) game between a user and an adversary that controls availability of channels. Given the user's strategy, the adversary turns on the subset of channels for which the user's gain is minimized. To offset this adversary, the user's objective is to design strategies which perform well with respect to all of the adversary's possible actions, i.e. in the worst-case. In 
subsequent sections, we develop the optimal strategy for the above problem, which is also shown to be a Nash equilibrium for this game. The corresponding worst-case performances (or equilibrium payoff) of these optimal strategies are also obtained.

The rest of this paper is organized as follows. Section II formulates the problem and primary worst-case performance metrics considered in this paper. Section III provides preliminary results, while Section IV presents a class of optimal strategies. Section $\mathrm{V}$ presents numerical results. In Section VI, the problem formulation and results are extended to analyze the case where probing may be imperfect. Section VII concludes the paper.

Unless otherwise stated, all results are proven in the Appendix. We have stated it can be shown for a few results that are easy to verify and thus not included due to space constraints.

\section{Problem Formulation}

In this section we formulate the problem considered in this paper. We then present various performance measures and corresponding objectives.

\section{A. Formulation and Strategies}

We consider a wireless system consisting of $N$ channels, indexed by the set $\Omega=\{1,2, \cdots, N\}$ and a transmitter who has access to these channels. The system works in discrete time, and at each time step the transmitter needs to determine which channels to use for transmission. To do so, the transmitter can probe up to $K$ of the $N$ channels, where $1 \leq K \leq N$.

A channel $j$ has a maximum data rate denoted by $r_{j}$. The actual data rate is given by a random variable $x_{j}$ that takes on any value between 0 and $r_{j}$. When the rate is positive (zero) then we say the channel is available/on (unavailable/off). If a channel $j$ is probed to be available and the transmitter decides to transmit in that channel, then it receives the rate $x_{j}$ (also referred to as the channel reward). If the channel is probed to be unavailable, then the transmitter is not allowed to use that channel for transmission. In addition, the transmitter is not allowed to transmit using unprobed channels.

The channel state is given by the random vector $\mathbf{x}=$ $\left[x_{1}, x_{2}, \cdots, x_{N}\right]$ where $P\left(0 \leq x_{j} \leq r_{j}\right)=1$. We denote by $X$ the set of all possible channel states. We assume that the transmitter knows the vector $\mathbf{r}=\left[r_{1}, r_{2}, \cdots, r_{N}\right]$. Without loss of generality, we will assume that $r_{1} \geq r_{2} \geq$ $\cdots \geq r_{N} \geq 0$. However, the transmitter does not have a priori information regarding the channel statistics, i.e., the probability distribution of $\mathbf{x}$. Thus the only information the transmitter has in making the probing decision is the maximum data rates of a channel.

The above assumptions are motivated by the channel access scenario in a cognitive radio network where a secondary user seeks to utilize portions of the spectrum not being used by a set of primary users. In doing so, it needs to find out which part of the spectrum is available for transmission; such availability is in general random depending on the primary users' traffic pattern. A secondary user may not be able to model this availability probabilistically, but it may know the maximum rate of a given channel when it is available due to information such as bandwidth, its modulation/coding schemes, propagation properties, etc.

In general, when the transmitter has no knowledge regarding channel availability, then $\mathbf{x} \in X$. We will also consider the case when somehow the transmitter knows the average number of available channels. It will be seen that having such knowledge can help the transmitter design better strategies. For this case, we define the following set of channel states:

$$
X_{L}=\left\{\mathbf{x} \in X \quad: E_{\mathbf{x}}\left[\sum_{j=1}^{N} I_{\left\{x_{j}>0\right\}}\right] \leq L\right\},
$$

where $E_{\mathbf{x}}$ denotes the expectation with respect to $\mathbf{x}$, and $I_{\{\cdot\}}$ is the indicator function. $X_{L}$ is the set of channel states such that, on average, at most $L$ channels are on. Note that $X_{N}=X$. For generality, we will derive results in this paper for general $L$, noting that by using $L=N$ our results directly apply to the case where the transmitter has no knowledge on the average number of available channels.

The system proceeds as follows. The transmitter decides which set of $K$ channels in $\Omega$ to probe. After probing, the transmitter then determines which $K_{0}$ of these $K$ probed channels to use for transmission. We describe a user's strategy as a (random) binary vector $\mathbf{u}=\left[u_{1}, u_{2}, \cdots, u_{N}\right]$, where $u_{j}=1$ means the user probes channel $j$; otherwise, $u_{j}=0$.

We denote the probability mass function of $u_{j}$ by $p_{u_{j}}$. Thus, $p_{u_{j}}(k)=P\left(u_{j}=k\right)$ for $k \in\{0,1\}$. Let $U$ be the set of strategies, fixed or randomized. We define the following set of strategies:

$$
U_{K}=\left\{\mathbf{u} \in U: P\left(\sum_{j=1}^{N} u_{j} \leq K\right)=1\right\} .
$$

That is, $U_{K}$ is the set of strategies that probe at most $K$ channels. Note that any strategy in $U_{K}$ must also satisfy: $\sum_{j=1}^{N} p_{u_{j}}(1)=E_{\mathbf{u}}\left[\sum_{j=1}^{N} I_{\left\{u_{j}=1\right\}}\right] \leq K$.

We will first assume the probing process is perfect; if probing indicates a channel is (not) available, then it is indeed (not) available. In Section VI we generalize results to imperfect probing, e.g. a channel could be unavailable (on) even if probing indicates that it is on (unavailable).

\section{B. Competitive Regret}

Our first worst-case performance measure is in the form of the difference with respect to a genie as we present below. We will use the following notation throughout this paper. First, we let $g_{k}(\mathbf{z})$ denote the sum of the $k$ largest elements of any vector $\mathbf{z}$ and for any integer $k$. In addition, we let $\mathbf{z}_{1} \cdot \mathbf{z}_{2}$ denote the dot product between any two vectors $\mathbf{z}_{1}$ and $\mathbf{z}_{2}$.

Now consider any strategy $\mathbf{u} \in U_{K}$. Letting $V_{\mathbf{x}}^{\mathbf{u}}$ denote its expected reward for any $\mathbf{x}$, we have $V_{\mathbf{x}}^{\mathbf{u}}=$ $E_{\mathbf{u}} E_{\mathbf{x}}\left[g_{K_{0}}(\mathbf{x} \cdot \mathbf{u})\right]$, because the transmitter will use the $K_{0}$ channels with highest rate that are probed and available. Now 
consider an omniscient observer or genie who knows the realization of channel states in advance and can use up to $K_{0}$ channels for transmission. For a given realization, the genie can simply use the $K_{0}$ available channels with highest bandwidth. Letting $V_{\mathbf{x}}^{*}$ denote its expected reward, we have $V_{\mathbf{x}}^{*}=E_{\mathbf{x}}\left[g_{K_{0}}(\mathbf{x})\right]$.

Taking the difference between the genie's expected reward and that of strategy $\mathbf{u}$, we obtain a comparison between the two. As the transmitter does not have any a priori information regarding $\mathbf{x}$, except possibly the average number of available channels, we take a maximum of this difference over all $\mathbf{x}$ to obtain the following worst-case performance measure of $\mathbf{u}: \delta^{\mathbf{u}}=\max _{\mathbf{x} \in X_{L}}\left\{V_{\mathbf{x}}^{*}-V_{\mathbf{x}}^{\mathbf{u}}\right\}$.

This performance measure $\delta^{\mathbf{u}}$ can be interpreted as the regret [8], or minimax regret [7] of $\mathbf{u}$. The quantity $\delta^{\mathbf{u}}$ provides an upperbound on the performance of strategy $\mathbf{u}$ relative to the best possible. That is, for any arbitrary state $\mathbf{x} \in X_{L}$, strategy $\mathbf{u}$ will obtain expected reward within $\delta^{\mathbf{u}}$ of the genie. We will refer to $\delta^{\mathbf{u}}$ as the worst-case reward (difference) of strategy $\mathbf{u}$.

$\delta^{\mathbf{u}}$ is often known as the competitive regret of $\mathbf{u}$ versus an oblivious adversary [7] who knows the distribution (but not realization) of $\mathbf{u}$ in advance. Given $\mathbf{u}$, the adversary chooses an $\mathbf{x} \in X_{L}$ that maximizes the difference between the reward of $\mathbf{u}$ and itself. An advantage of considering this competitive performance measure is that $\delta^{\mathbf{u}}$ provides a bound for the worst-case performance of strategy $\mathbf{u}$ over all $\mathbf{x}$. Therefore, we can obtain a performance guarantee for a given strategy even if there is no a priori knowledge on $\mathbf{x}$. On the other hand, this performance measure may be viewed as somewhat conservative since it calculates the performance over the worst $\mathbf{x}$, which may or may not be likely to occur. If more information is known about $\mathbf{x}$ then one can calculate the worst-case performance over a smaller set of $\mathbf{x}$ and obtain a more accurate performance guarantee. For further discussion of the advantages and disadvantages of the worstcase performance measure, see [7].

The objective for the first problem, which we call $(A)$, is to find the strategy which minimizes the above worst-case measure, i.e. obtain the following minimum:

$$
\delta^{*}=\min _{\mathbf{u} \in U_{K}} \max _{\mathbf{x} \in X_{L}}\left\{V_{\mathbf{x}}^{*}-V_{\mathbf{x}}^{\mathbf{u}}\right\},
$$

where we refer to $\delta^{*}$ as the minimum worst-case difference.

The mathematical abstraction and objective for (A) is similar to problem formulations of [9], [10] which were motivated by different applications. The paper [9] considers an inspector (corresponding to the transmitter in our problem) attempting to detect whether an inspectee has cheated by searching among a subset of the inspectee's bins or resources (corresponding to channels in a wireless system). The paper [10] also considers a related search problem where an individual must decide how to hide a fixed number of objects within a set of containers (corresponding to channels in (A)), each with equal capacity. It can be shown that both [9], [10] correspond to our problem scenario when all channels have an equal data rate, i.e. $r_{j}=r_{l}$ for all channels $j, l$, and when the adversary is allowed to transmit in all $N$ channels (compared to our formulation, which allows some $K \leq N$ ). Therefore the problem considered here is more general.

From the above description, we see that this problem can also be viewed as a two-player zero-sum game where the adversary's payoff is the regret and the transmitter's payoff is the negative of this. In Section IV-C, we show that the optimal strategy for (3) with a corresponding $\mathbf{x}$ form a Nash equilibrium of the game.

\section{Competitive Ratio}

Our second worst-case performance measure, also commonly used, is in the form of the ratio between the reward of a strategy and the genie. Consider the following worst-case performance measure of any strategy $\mathbf{u}: \rho^{\mathbf{u}}=$ $\min _{\mathbf{x} \in X_{L}} \frac{V_{\mathbf{x}}^{\mathbf{u}}}{V_{\mathbf{x}}^{*}}=\min _{\mathbf{x} \in X_{L}}\left\{\frac{E\left[g_{K_{0}}(\mathbf{x} \cdot \mathbf{u})\right]}{E\left[g_{K_{0}}(\mathbf{x})\right]}\right\}$, which is also known as the competitive ratio [7] of strategy $\mathbf{u}$. It is implied that the genie cannot choose all elements of $\mathrm{x}$ to be zero in order for the above ratio to be well defined. For any state $\mathbf{x}$, strategy $\mathbf{u}$ is guaranteed to obtain performance within a factor $\rho^{\mathbf{u}}$ of the best possible. Note that $\rho^{\mathbf{u}} \leq 1$ for any strategy $\mathbf{u}$, as it is impossible to do better than the genie. The corresponding objective for this problem, which we call $(B)$, is to determine the strategy achieving maximum worst-case performance, $\max _{\mathbf{u} \in U_{K}} \rho^{\mathbf{u}}$. Again this problem may be considered a two-player zero-sum game where the transmitter's payoff is given by the ratio.

\section{PRELIMINARIES}

We will assume that $r_{K}>0$. If this were not true, then it means there are less than $K$ channels with positive rate, and the optimal strategy is trivial: probe channel $j$ if $r_{j}>0$.

Lemma 1: Let $\tilde{L}=\min \left\{L, K_{0}\right\}$. For any $\mathbf{u} \in U_{K}, \delta^{\mathbf{u}}=$ $\max _{\mathbf{x} \in X_{\tilde{L}}}\left\{V_{\mathbf{x}}^{*}-V_{\mathbf{x}}^{\mathbf{u}}\right\}$.

The intuition behind this result is that the adversary who knows the channel state in advance needs only use $\tilde{L}$ channels. On the other hand, $V_{\mathbf{x}}^{\mathbf{u}}$ cannot decrease if more channels become available. For the remainder of this paper, we will let $\tilde{L}=\min \left\{L, K_{0}\right\}$. An important consequence of this result is that if $\mathbf{x} \in X_{\tilde{L}}$, then $V_{\mathbf{x}}^{*}-V_{\mathbf{x}}^{\mathbf{u}}=\sum_{j=1}^{N} E\left[x_{j}\right] p_{u_{j}}(0)$. We also have the following:

Lemma 2: For any $\tilde{L}$ and any strategy $\mathbf{u}$, we have: $\delta^{\mathbf{u}}=$ $\max _{\mathbf{x} \in \bar{X}_{\tilde{L}}}\left\{V_{\mathbf{x}}^{*}-V_{\mathbf{x}}^{\mathbf{u}}\right\}$, where $\bar{X}_{L}$ is the set of $\mathbf{x}$ in $X_{L}$ such that $P\left(x_{j} \in\left\{0, r_{j}\right\}\right)=1$ for all $j$.

In other words, this lemma states that the worst-case regret of any strategy can be calculated by only considering channel states where each channel is either available with the maximum rate $r_{j}$ or unavailable. The set of all such channel states in $X_{\tilde{L}}$ is denoted by $\bar{X}_{\tilde{L}}$ throughout this paper. Thus in calculating $\delta^{\mathbf{u}}$ for any strategy, we can replace $X_{L}$ with $\bar{X}_{\tilde{L}}$. Because all $\mathrm{x} \in \bar{X}_{\tilde{L}}$ are discrete random vectors, we will describe the marginal probabilities of any $\mathbf{x}$ by $p_{x_{j}}\left(r_{j}\right)=P\left(x_{j}=r_{j}\right)=1-P\left(x_{j}=0\right)$. 
These results simplify the calculation of $\delta^{\mathbf{u}}$ as follows:

$$
\begin{aligned}
\delta^{\mathbf{u}} & =\max _{\mathbf{x} \in \bar{X}_{\tilde{L}}} E_{\mathbf{u}} E_{\mathbf{x}}\left[\sum_{j=1}^{N} r_{j} I_{\left\{x_{j}=r_{j}, u_{j}=0\right\}}\right] \\
& =\max _{\mathbf{x} \in \bar{X}_{\tilde{L}}}\left\{\sum_{j=1}^{N} r_{j} \cdot p_{x_{j}}\left(r_{j}\right) \cdot p_{u_{j}}(0)\right\},
\end{aligned}
$$

where we have used the linearity of expectation and independence between $\mathbf{x}$ and $\mathbf{u}$. Note that $V_{\mathbf{x}}^{\mathbf{u}}, V_{\mathbf{x}}^{*}$, and $\delta^{\mathbf{u}}$ only depend on $\mathbf{u}$ and $\mathbf{x}$ through their marginal probabilities, and not through their joint probabilities. Therefore throughout this paper we will describe strategies and channel states in terms of their marginal probabilities ${ }^{1}$. In Section IV, we will demonstrate a method for constructing strategies based on these marginal probabilities.

We define the following parameter for any $K$ :

$$
M=\max \left\{n \in\{K, \cdots, N\}: r_{n}>0, r_{n} \geq \frac{(n-K)}{\sum_{j=1}^{n} \frac{1}{r_{j}}}\right\}
$$

Note that the above set is guaranteed to be nonempty, since $r_{K}>0$ and therefore $K$ lies in the above set. Also note that because $r_{j} \geq r_{k}$ whenever $j<k$, there is no division by 0 in the above summation. It will be seen that parameter $M$ is crucial to describing the optimal strategy, and for some special cases denotes the channels which should be probed.

We will assume without loss of generality that $N \geq$ $M+\tilde{L}$. If this condition does not hold, one can add extra channels with rates $r_{N+1}=\cdots=r_{N+\tilde{L}}=0$. As a user has no incentive to use these extra channels and they do not contribute to the total reward, they do not affect the optimal strategy or its expected reward. This assumption is made to avoid boundary conditions in describing strategies and their worst-case rewards, thus facilitating the description.

We introduce an additional notation as follows. For $M$ defined in (5), and any integer $\bar{M}$ such that $K \leq \bar{M} \leq M$, define: $\eta(\bar{M})=$ $\max \left\{m \in\{0, \cdots, \tilde{L}\}: r_{\bar{M}+m} \sum_{j=1}^{\bar{M}} \frac{1}{r_{j}} \geq \bar{M}-K\right\}$, where $\eta(\bar{M})=0$ if this set is empty. This term $\eta(\bar{M})$ is introduced simply for notational purposes in describing the following class of algorithms and their worst-case rewards:

Definition 1: For any set of channels, define $M$ from (5). Then for any integer $K$, strategy $\mathbf{u}[l, \bar{M}]$ is defined by configurable parameters (integers) $l$ and $\bar{M}$, where $K \leq$ $\bar{M} \leq M$ and either $l=0$ or $\eta(\bar{M})+1 \leq l \leq \eta(\bar{M}-1)-1$. The marginal probabilities under $\mathbf{u}[l, \bar{M}]$ depend on $l$ and $\bar{M}$ as follows:

If $l=0$, the marginal probabilities under $\mathbf{u}[l, \bar{M}]$ are as follows. If $1 \leq i \leq \bar{M}$, let $p_{u_{i}}(1)=1-\frac{\bar{M}-K}{r_{i} \sum_{j=1}^{M} \frac{1}{r_{j}}}$. Otherwise, $p_{u_{i}}(1)=0$ if $\bar{M}+1 \leq i \leq N$.

If $\eta(\bar{M})+1 \leq l \leq \eta(\bar{M}-1)-1$, the marginal probabilities

\footnotetext{
${ }^{1}$ In [9] (Section 4) it was shown that for every set of marginal probabilities there corresponds a strategy $\mathbf{u}$. Thus, if we can determine a set of optimal marginal probabilities in 4 , then there must be an optimal strategy in $U$ with these marginal probabilities.
}

under $\mathbf{u}[l, \bar{M}]$ are defined as follows. If $1 \leq i \leq \bar{M}-1$, then $p_{u_{i}}(1)=1-\frac{r_{\bar{M}+l}}{r_{i}}$. If $i=\bar{M}$, then $p_{u_{i}}(1)=1-\bar{M}+K+$ $r_{\bar{M}+l} \sum_{j=1}^{\bar{M}-1} \frac{1}{r_{j}}$. Otherwise, $p_{u_{i}}(1)=0$ for $\bar{M}+1 \leq i \leq N$.

The parameters $l$ and $\bar{M}$ essentially determine a randomized strategy. Note that from the definitions of $M$ and $\eta$ the strategy $\mathbf{u}[l, \bar{M}]$ has valid marginal probabilities for the range of $l$ and $\bar{M}$ specified in Definition 1 . When $l=0$, then by definition of $M$ and $K \leq \bar{M} \leq M$, we have $0 \leq p_{u_{i}}(1) \leq 1$ for all $i$. For $l \neq 0$, we can also show that $\mathbf{u}[l, \bar{M}]$ is a valid strategy as follows. Since $r_{\bar{M}+l} \leq r_{\bar{M}}$ for any $l \geq 1$, then we only need to show that $0 \leq p_{u_{\bar{M}}}(1) \leq 1$. First, $l \leq \eta(\bar{M}-1)-1$ implies from the definition of $\eta$ that $r_{\bar{M}-1+l+1} \sum_{j=1}^{\bar{M}-1} \frac{1}{r_{j}} \geq \bar{M}-1-K$. Rearranging this yields $0 \leq p_{u_{\bar{M}}}(1)$. Similarly, $l \geq \eta(\bar{M})+1$ gives: $r_{\bar{M}+l} \sum_{j=1}^{\bar{M}} \frac{1}{r_{j}}<\bar{M}-K$. Rearranging this and using $\frac{r_{\bar{M}+l}}{r_{M}}>0$ yields $p_{u_{\bar{M}}}(1)<1$, thus completing the proof that $\mathbf{u}[l, M]$ has valid marginal probabilities for $l \neq 0$.

We provide some intuition for considering the above class of strategies via an example. Suppose $M=N$ and consider strategy $u[0, N]$. We see that the term $p_{u_{j}}(0) r_{j}$ is constant for all $1 \leq j \leq N$. From (4), this means that the worstcase difference for $\mathbf{u}$ only depends on the sum of the marginal probabilities of $\mathbf{x}$, but not the individual marginal probabilities. Thus, $\mathbf{u}[0, M]$ has constant performance for different $\mathbf{x}$. This type of constant performance with respect to $\mathbf{x}$ is a common trait of robust worst-case strategies [7]. Similar reasoning can be applied to $\mathbf{u}[l, \bar{M}]$ for other values of $l$ and $\bar{M}$. For these strategies, the term $p_{u_{j}}(0) r_{j}$ is constant for $1 \leq j \leq \bar{M}$.

In the next section, we will show that the optimal strategy for the competitive regret belongs to the class of strategies given by Definition 1 .

\section{Optimal Strategies}

In this section, we present optimal strategies for $(A)$ and $(B)$. To facilitate the description, we define the following for any $m \geq K: \gamma_{m}=\frac{\tilde{L}-\eta(m)}{\sum_{j=1}^{m} \frac{1}{r_{j}}}$.

\section{A. Optimal Strategies for $(A)$}

For any fixed $K$, we let $W_{K}=\cup_{\bar{M}} \cup_{l} \mathbf{u}[l, \bar{M}]$ be the set of all strategies given by Definition 1 . We now describe the optimal strategy for $(A)$ :

Theorem 1: Optimal Strategy for $(A)$ For any set of channels, define $M$ from (5). Then the optimal strategy $\mathbf{u}^{*} \in W_{K}$, i.e. $\min _{\mathbf{u} \in U_{K}} \delta^{\mathbf{u}}=\min _{\mathbf{u} \in W_{K}} \delta^{\mathbf{u}}$, and is determined as follows.

1) If there exists $K \leq M^{*} \leq M$ satisfying $r_{M^{*}} \geq$ $\gamma_{M^{*}} \geq r_{M^{*}+1} I_{\left\{M^{*}<M\right\}} \quad$, then $\mathbf{u}\left[0, M^{*}\right]$ is the optimal strategy.

2) Otherwise, there must exist an $M^{*}$, where $K+$ $1 \leq M^{*} \leq M$, satisfying the following inequality: $\gamma_{M^{*}}>r_{M^{*}} \geq \gamma_{M^{*}-1}$. Then the optimal strategy is $\mathbf{u}\left[l^{*}, M^{*}\right]$, where: $l^{*}=$ $\min \left\{m \in\{0, \cdots, \tilde{L}\}: m \geq \tilde{L}-r_{M^{*}} \sum_{j=1}^{M^{*}} \frac{1}{r_{j}}\right\}$. 
Theorem 1 is proved in Section IV-C. Also, the corresponding worst-case rewards (which are the minimum obtainable) are given in Lemma 4 in closed form. In Section IV-C we provide a game-theoretic interpretation of Theorem 1 .

Note that Theorem 1 provides a method to determine the optimal strategy, and this procedure is a fairly simple one. In particular, cases 1 and 2 each require checking whether a condition holds, and there are only $M-K+1$ possibilities for the value of $M^{*}$. The term $\eta(\cdot)$ is also not difficult to determine. An alternative method for finding the optimal strategy is as follows. First, note that $W_{K}$ contains at most $(M-K+1) \tilde{L}$ strategies. For each $\mathbf{u}[l, \bar{M}] \in W_{K}$, the worstcase difference $\delta^{\mathbf{u}[l, M]}$ can be easily determined, as we will show in Lemma 4 in Section IV-C. Thus combining this with $\left|W_{K}\right| \leq(M-K+1) \tilde{L}$, the best strategy in $W_{K}$ can be determined with low computational complexity.

We provide some intuition for Theorem 1. In both cases 1 and 2 of the above theorem, there are $M^{*}$ channels with rewards significantly greater than other channels, and only these channels should be probed. The probability distribution assigned to probing each channel depends on the relationship between $\tilde{L}, K$ and $M$. An interesting special case of the above result is the following. When $K+\tilde{L} \leq M$, then from the definition of $M$, case 1 of Theorem 1 holds with $M^{*}=M$. Thus we have the following corollary:

Corollary 1: For any set of channels, define $M$ from (5). Then if $K+\tilde{L} \leq M$, the optimal strategy $\mathbf{u}^{*}$ has the same marginal probabilities as strategy $\mathbf{u}[0, M]$ given by Definition 1 .

Note that Corollary 1 and Theorem 1 describe the optimal marginal probabilities, but they do not immediately or uniquely determine an optimal strategy. By definition of $U_{K}$, at most $K$ channels can be probed for any realization of $\mathbf{u}$. Thus, it cannot be possible that channel selection is done independently for each channel. We could compute the joint probabilities of these random vectors of length $N$ by solving a system of linear equations (with $N$ equations and up to $2^{N}$ unknowns). However, the number of length $N$ binary vectors with exactly $K$ ones grows very quickly as $N$ increases.

Below we present a procedure (Algorithm 1) that computes $\mathbf{u}[0, M]$ in a sequential manner without considering the entire set of binary vectors of length $N$.

Algorithm 1: (Sequential Method to Construct $\mathbf{u}[0, \bar{M}]$ ) For any $N$ and $K$, define $M$ as in (5) and consider any $K \leq \bar{M} \leq M$.

Initially: $\bar{M}, K$ are defined. Set $j=1$ and $u_{l}=0$ for all $1 \leq l \leq N$.

Step 1: Let $p=1-\frac{\bar{M}-K}{r_{j} \sum_{l=1}^{M} \frac{1}{r_{l}}}$. Update $K$ and $\mathbf{u}$ as follows:

- With probability $p$, set $u_{j}=1$ and $K=K-1$.

- Otherwise (with probability $1-p$ ), do not change $\mathbf{u}, K$.

Step 2: If $K=0$, stop and use strategy u. Otherwise, repeat Step 1 with $j=j+1, \bar{M}=\bar{M}-1$, and using the updated $\mathbf{u}$ and $K$.

This algorithm takes inputs $N, K$ and sequentially generates $\mathbf{u}[0, \bar{M}]$. We note from Step 2 of this description that
Algorithm 1 constructs a strategy $\mathbf{u}$ which, with probability 1 , probes at most $K$ channels. Thus, $\mathbf{u} \in U_{K}$. We have the following result on Algorithm 1.

Lemma 3: Algorithm 1 constructs a strategy with marginal probabilities which match the marginal probabilities of $\mathbf{u}[0, \bar{M}]$ given by Definition 1 .

Therefore, by using $\bar{M}=M$ in Algorithm 1, we can construct the optimal strategy given in Corollary 1. In Section $\mathrm{V}$, we will show that $\mathbf{u}[0, M]$ performs very well compared to the optimal strategy given by Theorem 1. Thus, strategy $\mathbf{u}[0, M]$ is a practical alternative if the optimal strategy of Theorem 1 is difficult to construct. Algorithm 1 also constructs strategy $\mathbf{u}[0, \bar{M}]$ for any $K \leq \bar{M} \leq M$; thus it generates the optimal strategy whenever case 1 of Theorem 1 holds. For $l>0$, it is not immediately clear whether there exists a sequential algorithm to easily construct $\mathbf{u}[l, \bar{M}]$. This is a subject of further study.

\section{B. Optimal Strategies for $(B)$}

For Problem $(B)$ we have the following result:

Theorem 2: Consider any set of channels and integers $L, K$. We have the following result for $(B): \max _{\mathbf{u} \in U_{K}} \rho^{\mathbf{u}}=$ $\max _{\mathbf{u} \in U_{K}} \bar{\rho}^{\mathbf{u}}=K / N$, where the optimal strategy $\mathbf{u}^{*}$ achieving this maximum reward ratio has marginal probabilities given by: $p_{u_{j}^{*}}(1)=K / N$ for all $1 \leq j \leq N$.

We see from Theorem 2 there exists an optimal strategy which uniformly chooses from all length- $N$ binary vectors with exactly $K$ elements equal to 1 . In Section $\mathrm{V}$, we compare the performance of this uniform strategy to the optimal strategies of Theorem 1.

\section{Deriving Optimal Strategies}

To prove Theorem 1, we first derive results on the set of strategies of Definition 1.

Lemma 4: For any set of channels, consider any strategy $\mathbf{u}[l, \bar{M}]$ given by Definition 1 .

If $l=0$, then $\delta^{\mathbf{u}[0, \bar{M}]}=\sum_{j=1}^{\eta(\bar{M})} r_{\bar{M}+j}+(\bar{M}-K) \gamma_{\bar{M}}$ Otherwise, for $\eta(\bar{M})+1 \leq l \leq \eta(\bar{M}-1)-1$, we have: $\delta^{\mathbf{u}[l, \bar{M}]}=\left(\bar{M}-K-r_{\bar{M}+l} \sum_{j=1}^{\bar{M}-1} \frac{1}{r_{j}}\right) r_{\bar{M}}+\sum_{k=1}^{l-1} r_{\bar{M}+k}+$ $(\tilde{L}-l) r_{\bar{M}+l}$.

Now we prove that these strategies are optimal. It can be shown (see [11]) that by interchanging maximum and minimum, the following is always true:

$$
\min _{\mathbf{u} \in U_{K}} \max _{\mathbf{x} \in X_{L}}\left\{V_{\mathbf{x}}^{*}-V_{\mathbf{x}}^{\mathbf{u}}\right\} \geq \max _{\mathbf{x} \in X_{L}} \min _{\mathbf{u} \in U_{K}}\left\{V_{\mathbf{x}}^{*}-V_{\mathbf{x}}^{\mathbf{u}}\right\}
$$

The inequality in (6) is useful for deriving lowerbounds to $\delta^{*}$ as follows. Note that for any $\mathbf{x}$, the righthand-side of (6) is an optimization problem with objective to minimize an average-reward criterion. By choosing $\mathbf{x} \in X_{L}$ such that the optimal average-reward is high, we can obtain a useful lowerbound to the minimax regret. We then show that these lower bounds match the quantities given in Lemma 4, thereby proving the optimality of $\mathbf{u}\left[0, M^{*}\right]$ for some $M^{*}$.

We first prove case 1 of Theorem 1. To derive a lowerbound in (6), consider the following: 
Problem 1: For any given $\tilde{L}, K$ and set of channels, define $M$ as in (5). Suppose case 1 of Theorem 1 holds for some $M^{*}$, and let state $\mathrm{x}$ have the following marginal probabilities. If $1 \leq i \leq M^{*}$, then set $p_{x_{i}}\left(r_{i}\right)=\frac{\gamma_{M^{*}}}{r_{i}}$. If $M^{*}+1 \leq i \leq M^{*}+\eta\left(M^{*}\right)$, let $p_{x_{i}}\left(r_{i}\right)=1$. Otherwise, for all other values of $i$, let $p_{x_{i}}\left(r_{i}\right)=0$.

Solution: The optimal strategy for this problem will probe any $K$ of the channels in set $\left\{1, \cdots, M^{*}\right\}$ (either randomly or deterministically), and satisfies: $\min _{\mathbf{u} \in U_{K}}\left\{V_{\mathbf{x}}^{*}-V_{\mathbf{x}}^{\mathbf{u}}\right\}=$ $\delta^{\mathbf{u}\left[0, M^{*}\right]}$

This result is proven in Appendix F. Combining the result of Problem 1 with (6), we see that $\mathbf{u}\left[0, M^{*}\right]$ must be the optimal worst-case strategy.

Now we show that when case 1 of Theorem 1 does not hold, then case 2 must be true. When case 1 of this theorem is not true for all $K \leq \bar{M} \leq M$, then for each $\bar{M}$ one of the following is true: either $r_{\bar{M}} \sum_{j=1}^{\bar{M}} \frac{1}{r_{j}}<\tilde{L}-\eta(\bar{M})$ or $r_{\bar{M}+1} \sum_{j=1}^{\bar{M}} \frac{1}{r_{j}}>\tilde{L}-\eta(\bar{M})$. Combining this with $\eta(K)=\tilde{L}$ from the definition of $\eta$, it can be shown that case 2 must be satisfied for some $M^{*}$.

Now consider $l^{*}$ and $M^{*}$ as described by case 2 of Theorem 1 . To prove that $\mathbf{u}\left[l^{*}, M^{*}\right]$ as described by case 2 is optimal, we consider the following problem.

Problem 2: For any given $\tilde{L}, K$ and set of channels, define $M$ as in (5). Suppose case 2 of Theorem 1 holds for some $M^{*}$ and $l$. Let state $\mathrm{x}$ have the following marginal probabilities: For $1 \leq i \leq M^{*}-1$, let $p_{x_{i}}\left(r_{i}\right)=\frac{r_{M^{*}}}{r_{i}}$. For $M^{*} \leq i \leq M^{*}+l-1$, let $p_{x_{i}}\left(r_{i}\right)=1$. When $i={\stackrel{r_{i}}{M}}^{*}+l$, then let $p_{x_{i}}\left(r_{i}\right)=\tilde{L}-l-r_{M^{*}} \sum_{j=1}^{M^{*}-1} \frac{1}{r_{j}}$. Otherwise, for all other values of $i$, let $p_{x_{i}}\left(r_{i}\right)=0$.

Solution: As shown in Appendix G, the optimal strategy for this problem probes any $K$ of the channels in set $\left\{1, \cdots, M^{*}\right\}$ and satisfies: $\min _{\mathbf{u} \in U_{K}}\left\{V_{\mathbf{x}}^{*}-V_{\mathbf{x}}^{\mathbf{u}}\right\}=$ $\delta^{\mathbf{u}\left[l, M^{*}\right]}$.

Combining this with (6) proves that $\mathbf{u}\left[l, M^{*}\right]$ must be optimal for case 2 of Theorem 1.

We now provide a game theoretic interpretation to the results for Theorem 1. Consider the equivalent two-player zero-sum game mentioned earlier, with a user who chooses $\mathbf{u}$ and an adversary that selects $\mathbf{x}$. We have proven that $\max _{\mathbf{x} \in X_{L}} \min _{\mathbf{u} \in U_{K}}\left\{V_{\mathbf{x}}^{*}-V_{\mathbf{x}}^{\mathbf{u}}\right\}=$ $\min _{\mathbf{u} \in U_{K}} \max _{\mathbf{x} \in X_{L}}\left\{V_{\mathbf{x}}^{*}-V_{\mathbf{x}}^{\mathbf{u}}\right\}$. From [12] (Proposition 22.2), this means that $\mathbf{u}^{*}$ from Theorem 1 and the $\mathbf{x}^{*}$ for Problems 1 and 2 form a Nash equilibrium for this strictly competitive game. A similar interpretation holds for the competitive ratio and optimal worst-case strategy in Theorem 2 .

\section{Numerical Results}

In this section, we examine the performance of the proposed algorithms under a range of system parameters. We first compare the performance between the optimal strategies under the different metrics described in Section II. Then we compare the worst-case strategies to other heuristics or algorithms.

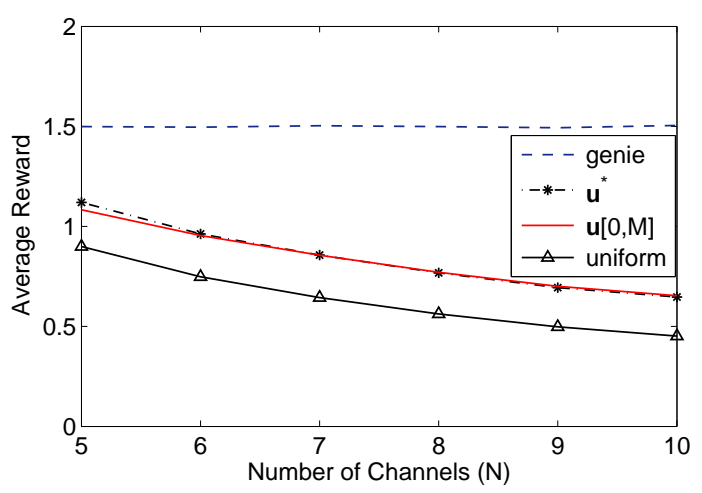

Fig. 1. Average performance of the genie, algorithms $\mathbf{u}^{*}, \mathbf{u}[0, M]$, and uniform as described in Section $\mathrm{V}$, when $K=L=3$ and $N$ varies from 5 to 10

\section{A. Comparison of Metrics}

For the numerical results, we chose parameters $r_{j}$ as follows. For a given $N$, the $r_{j}$ were uniformly distributed in $[0,1]$. For each realization of the rates $\left\{r_{j}\right\}_{j \in S}$, we computed the average reward obtained by the following four strategies: (1) genie, or omniscient observer: knows in advance the $\tilde{L}$ available channels with highest bandwidth (2) $\mathbf{u}^{*}$ : optimal strategy within the class given by Definition 1 , and shown to be optimal in Theorem 1 (3) $\mathbf{u}[0, M]$ : the optimal strategy under the conditions given by Corollary 1 and (4) uniform: randomly probe $K$ out of the $N$ channels, optimal for (B) in Theorem 2.

Figure 1 shows the performance of these two strategies when we fix $K=L=3$ and $N$ varies from 5 to 10 . In order to achieve $L=3$, we set $p_{x_{j}}\left(r_{j}\right)=3 / N=1-p_{x_{j}}(0)$ for all $1 \leq j \leq N$. We see that $\mathbf{u}^{*}$ outperforms $\mathbf{u}[0, M]$ and uniform for most $N$. Note that for large $N, \mathbf{u}[0, M]$ slightly outperforms $\mathbf{u}^{*}$. This is because even though $\mathbf{u}^{*}$ may have a lower worst-case difference (bound over all $\mathbf{x}$ ), it does not necessarily outperform $\mathbf{u}[0, M]$ for some given $\mathbf{x}$. In general, the performance of these two strategies is similar for most $N$, especially as $N$ increases. The latter can be explained by the fact that as $N$ (and $M$ ) increase while $K$ stays fixed, it becomes more likely that the conditions of Corollary 1 are satisfied. Hence, as $N$ increases then it is more likely that $\mathbf{u}^{*}$ and $\mathbf{u}[0, M]$ are the same strategy. The performance of the genie varies for each realization of the $\left\{r_{j}\right\}$ and $\mathbf{x}$, but its average performance is relatively constant with respect to $N$ since $L=3$ is fixed and the genie only uses the $K=3$ available channels with highest rate.

Figure 2 shows the performance of these strategies when $N=10, L=5$ and $K$ increases from 3 to 9 . As expected, the average rewards of $\mathbf{u}^{*}, \mathbf{u}[0, M]$, and uniform all approach the genie's average reward as $K$ increases, because it becomes more likely these strategies will probe the best channels as $K$ increases. Meanwhile, $\mathbf{u}^{*}$ strictly outperforms uniform for all $K$.

These results indicate that the optimal strategies under the worst-case difference performance measure appear to outperform the optimal algorithm (uniform) arising from the 


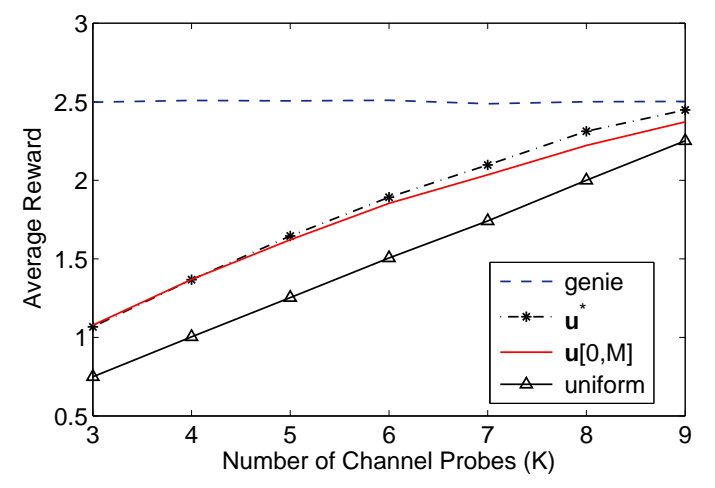

Fig. 2. When $N=10$ and $L=5$, average performance of strategies as $K$ increases from 3 to 9 .

worst-case ratio performance measure. In addition, if the procedure of determining $\mathbf{u}^{*}$ becomes impractical, then one can instead use $\mathbf{u}[0, M]$ which has comparable performance for the scenario described in this section but is very easy to construct using the proposed Algorithm 1 of Section IV-A.

\section{B. Sensitivity Analysis of Strategies}

The optimal strategies in Section IV are derived under worst-case performance measures. If the transmitter knows the distribution of $\mathbf{x}$, i.e. channel states, then it can probe and use the $K$ channels with highest values of $p_{x_{i}}\left(r_{i}\right) r_{i}$ in order to maximize $V_{\mathbf{x}}^{\mathbf{u}}$ (we call this the optimal average-reward strategy) and outperform the optimal worst-case strategies. However, if this knowledge of the distribution is incorrect then the optimal average-reward strategy may have a poor performance. The optimal worst-case strategies, by contrast are guaranteed to perform well over all $\mathrm{x}$ and could be more robust to changes in $\mathbf{x}$.

To illustrate this, we compare various strategies as follows, where $N=10$ and $L=K=5$. For any given set of rewards $\left\{r_{j}\right\}, p_{x_{j}}\left(r_{j}\right)=j^{\alpha} / \beta=1-p_{x_{j}}(0)$ for all $1 \leq j \leq N$ and some $-5 \leq \alpha \leq 5$. Here, $\beta$ is a normalizing constant to ensure that $\mathrm{x} \in X_{L}$. Note that when $\alpha=0$, then $\mathbf{x}$ takes on the uniform distribution. Negative (positive) $\alpha$ means the channels with higher (lower) rate have a higher probability of being available. We let $\mathbf{v}_{0}$ and $\mathbf{v}_{2}$ to be the optimal averagereward strategies when the transmitter believes $\alpha=0,2$, respectively. Note that strategy $\mathbf{v}_{0}$ (or $\mathbf{v}_{2}$ ) probes the $K$ channels with the highest values of $r_{j}$ (or $\left.r_{j} j^{2} / \beta\right)$. Thus, these strategies can be seen as simple heuristics that make probing decisions based on weighted values of the channel rates.

As shown in Figure 3, we examined the performance of these strategies by varying the real value of $\alpha$. For a given $\alpha$, the $\left\{r_{j}\right\}$ were uniformly distributed in $[0.8,1.2]$ and the average performance of $\mathbf{v}_{0}, \mathbf{v}_{2}, \mathbf{u}^{*}, \mathbf{u}[0, M]$, and uniform was determined for $10^{4}$ realizations. This process was repeated for $-5 \leq \alpha \leq 5$. Note that performance of $\mathbf{v}_{0}$ and $\mathbf{v}_{2}$ under various $\alpha$ indicates the effect of errors in the transmitter's belief of the true $\alpha$.

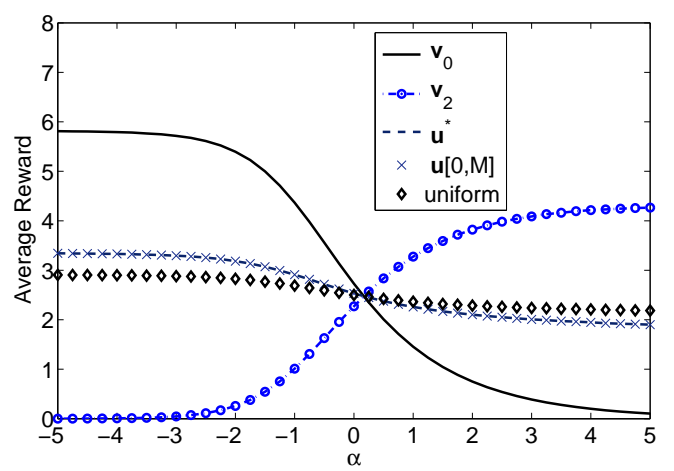

Fig. 3. Comparison of optimal average-reward strategies $\mathbf{v}_{0}$ and $\mathbf{v}_{2}$ to the optimal worst-case strategies $\mathbf{u}^{*}, \mathbf{u}[0, M]$ and uniform as the channel state distribution is varied, as described in Section V-B.

We see that for negative (positive) $\alpha, \mathbf{v}_{0}\left(\mathbf{v}_{2}\right)$ performs very well. On the other hand, we see that as $\alpha$ increases (decreases), the expected reward of $\mathbf{v}_{0}\left(\mathbf{v}_{2}\right)$ approaches zero. This is because $\mathbf{v}_{0}$ probes the channels with the $K$ highest rewards, thus it performs better when the channels with higher rate have a higher probability of being available. Similar reasoning can be applied for $\mathbf{v}_{2}$. On the other hand, $\mathbf{u}^{*}$ and $\mathbf{u}[0, M]$ are relatively robust to changes in the value of $\alpha$, as their total reward does not approach 0 if $\alpha$ varies. This example illustrates the robustness of worstcase strategies compared to optimal average-reward strategies under varying distributions.

\section{Probing ERrors}

The problem formulated in Section II assumes that if the probing results indicate a channel is (not) available, then it is indeed (not) available. In this section, we extend the results of the previous sections to imperfect probing. For notational convenience, we present results here for $\mathbf{x} \in \bar{X}_{L}$, noting that our results can be generalized to $X_{L}$ similar to Lemma 2 .

We consider the following modified version of the problem outlined in Section II. For each channel $i$, let $y_{i}=1$ if channel $i$ is probed to be available, and $y_{j}=0$ if probing indicates the channel is off. Probing results are collectively described by probing result vector $\mathbf{y}=\left[y_{1}, \cdots, y_{N}\right]$. Since the transmitter can only probe up to $K$ channels, it will only learn $K$ elements of vector $\mathbf{y}$; the other $N-K$ values remain unknown.

When a probe reveals that a channel is available, then with probability $p$ the channel is actually available. Equivalently, defining $\mathbf{x}$ as in Section II, $P\left(x_{j}>0 \mid y_{j}=1\right)=p$. We assume the probing errors occur independently among different channels, i.e. $P\left(x_{k}>0 \mid y_{j}=1\right)=P\left(x_{k}>0\right)$ if $k \neq j$. Similarly, a channel probed to be unavailable is actually available with probability $q$, i.e. $P\left(x_{j}>0 \mid y_{j}=0\right)=q$. These conditions imply $q \leq P\left(x_{j}>0\right) \leq p$. Note that $p=1$ and $q=0$ describes the original problem formulation considered in the previous sections. The values of $p$ and $q$ may depend on the physical sensor [2], and are assumed to be known in advance to the transmitter. We assume without 
loss of generality that $p>q$; if this assumption does not hold, then one can easily reverse the definition of the probing results to obtain $p>q$. We will assume that $p$ and $q$ are the same for all channels.

We note that from the marginal probabilities of $\mathbf{y}$, one can determine the marginal probabilities of any channel being available. Specifically, $p_{x_{j}}\left(r_{j}\right)=P\left(x_{j}=r_{j} \mid y_{j}=\right.$ 1) $\cdot p_{y_{j}}(1)+P\left(x_{j}=r_{j} \mid y_{j}=0\right) \cdot p_{y_{j}}(0)=(p-q) \cdot p_{y_{j}}(1)+$ $q$. Similarly, one can uniquely determine $p_{y_{j}}(1)$ from $p_{x_{j}}\left(r_{j}\right)$. Thus, in this section we will describe the system state in terms of vector $\mathbf{y}$ while noting that our results hold for a unique corresponding random vector $\mathbf{x}$. For any $\mathbf{y}$, strategy $\mathbf{u}$ has expected reward $V_{\mathbf{y}}^{\mathbf{u}}=$ $E_{\mathbf{u}} E_{\mathbf{y}}\left[g_{K_{0}}(p \cdot \mathbf{r} \cdot \mathbf{y} \cdot \mathbf{u}+q \cdot \mathbf{r} \cdot(1-\mathbf{y}) \cdot \mathbf{u})\right]$, where $(1-\mathbf{y})$ denotes the vector $\left[1-y_{1}, 1-y_{2}, \cdots, 1-y_{N}\right]$, because the transmitter will use for transmission the $K_{0}$ probed channels with highest value of either $p \cdot r_{j}$ or $q \cdot r_{j}$, depending on the probing results. Similarly, a genie who knows the probing results $\mathbf{y}$ in advance (but not $\mathbf{x}$ ) will attain expected reward $V_{\mathbf{y}}^{*}=E_{\mathbf{y}}\left[g_{K_{0}}(p \cdot \mathbf{r} \cdot \mathbf{y}+q \cdot \mathbf{r} \cdot(1-\mathbf{y}))\right]$.

The objective for this problem, which we call (A'), is to determine the strategy minimizing the worst-case difference: $\max _{\mathbf{y} \in Y_{L}}\left\{V_{\mathbf{y}}^{*}-V_{\mathbf{y}}^{\mathbf{u}}\right\}$, where $Y_{L}$ is defined analogously to $X_{L}$ in Section II. Strategies minimizing this worst-case difference will be described as optimal strategies for ( $\left.A^{\prime}\right)$. Analogous to equation (5), we define the following constant: $M=\max \left\{n \in\{K, \cdots, N\}: r_{n}>0, r_{n} \geq \frac{(n-K) p+K q}{p \sum_{k=1}^{n} \frac{1}{r_{k}}}\right\}$, where $M=K$ if this set is empty. Similarly, for any $K \leq \bar{M} \leq M$, define function $\eta(\cdot)$ as follows: $\eta(\bar{M})=\max \left\{m \in\{0, \cdots, \tilde{L}\}: r_{\bar{M}+m} \geq \frac{(\bar{M}-K) p+q K}{p \sum_{j=1}^{M} \frac{1}{r_{j}}}\right\}$, where again $\tilde{L}=\min \left\{L, K_{0}\right\}$

Then we have the following result on the optimal strategy for $\left(\mathrm{A}^{\prime}\right)$ under certain conditions:

Theorem 3: Consider any set of channels, integers $\tilde{L}, K$, and define $M$ as above. If the following inequality holds: $r_{M^{*}} \geq \frac{\left[\tilde{L}-\eta\left(M^{*}\right)\right] p+q\left(M^{*}-\tilde{L}+\eta\left(M^{*}\right)\right)}{p \sum_{k=1}^{M^{*}} \frac{1}{r_{k}}} \geq r_{M^{*}+1} I_{\left\{M^{*}<M\right\}}$ for some $K \leq M^{*} \leq M$, and if $r_{1}$ satisfies: $q \cdot r_{1} \sum_{k=1}^{M^{*}} \frac{1}{r_{k}} \leq$ $\left(M^{*}-K\right) p+q \bar{K}$, then the optimal strategy for (A') has the following marginal probabilities: $p_{u_{j}}(1)=1-$ $\frac{\left(M^{*}-K\right) p+K q}{(p-q) r_{j} \sum_{k=1}^{M^{*}} \frac{1}{r_{k}}}+\frac{q}{p-q}$ for $1 \leq j \leq M^{*}$, and $p_{u_{j}}(1)=0$ for $j \geq M^{*}+1$.

Note that plugging in $p=1$ and $q=0$ into the above theorem yields then we obtain Case 1 of Theorem 1 . Generalizing the above result for Case 2 of Theorem 1 is part of the future work. In addition, Algorithm 1 of Section IV-A can be modified to construct the optimal strategy in Theorem 3 , by replacing $\tilde{p}$ in the algorithm with $p_{u_{j}}(1)$ of this theorem. Thus, the above optimal strategy can be constructed in a sequential manner, with low computational complexity.

\section{CONCLUSION}

We examined optimal competitive algorithms for joint channel probing and transmission. We formulated multiple worst-case performance measures and derived a class of optimal strategies. We presented an algorithm which sequentially generates a subclass of these strategies with low computational complexity. The performances of these strategies were also examined via numerical studies. These results and algorithms are applicable to many practical scenarios, particularly when the channel quality is changing unpredictably.

\section{REFERENCES}

[1] J. Kennedy and M. Sullivan, "Direction finding and "smart antennas" using software radio architectures," IEEE Communications Magazine, pp. 62-68, May 1995.

[2] Y. Chen, Q. Zhao, and A. Swami, "Joint design and separation principle for opportunistic spectrum access," Proceedings of IEEE Asilomar Conference on Signals, Systems, and Computers, November 2006, Pacific Grove, CA

[3] G. Holland, N. Vaidya, and P. Bahl, "A rate-adaptive mac protocol for multi-hop wireless networks," Proceedings of the Seventh International Conference on Mobile Computing and Networking (MOBICOM), 2001, Rome, Italy.

[4] A. Sabharwal, A. Khoshnevis, and E. Knightly, "Opportunistic spectral usage: bounds and a multi-band CSMA/CA protocol," IEEE/ACM Transactions on Networking, pp. 533-545, June 2007.

[5] S. Guha, K. Munagala, and S. Sarkar, "Approximation schemes for information acquisition and exploitation in multichannel wireless networks," Proceedings of Fourty-Fourth Annual Allerton Conference on Communication, Control and Computing, September 2006, Monticello, IL.

[6] N. Chang and M. Liu, "Optimal channel probing and transmission scheduling for opportunistic spectrum access," Proceedings of the Thirteenth International Conference on Mobile Computing and Networking (MOBICOM), September 2007, Montreal, CN.

[7] A. Borodin and R. El-Yaniv, Online Computation and Competitive Analysis, Cambridge University Press, 1998, Cambridge, UK.

[8] D. Foster and R. Vohra, "Regret in the on-line decision problem," Games and Economic Behavior, vol. 29, pp. 1084-1090, 1999.

[9] A.J. Goldman and M.H. Pearl, "The dependence of inspection-system performance on levels of penalties and inspection resources," Journal of Research of the National Bureau of Standards, vol. 80B, 1976.

[10] N.Zoroa, M.J. Fernandez-Saez, and P. Zoroa, "Search and ambush games with capacities," Journal of Optimization Theory and Applications, vol. 123, no. 2, pp. 431-450, 2004.

[11] S. Nash and A. Sofer, Linear and Nonlinear Programming, McGrawHill, 1996, New York, USA.

[12] M. Osborne and M. Rubinstein, A Course in Game Theory, The MIT Press, 1994, Cambridge, MA.

[13] N.Chang and M.Liu, "Competitive analysis of opportunistic spectrum access strategies," Proceedings of the Twenty-Seventh IEEE Conference on Computer Communications (INFOCOM), April, 2008, Phoenix, AZ.

\section{APPENDIX}

We note that the proofs in Appendices C,E,F,G have appeared in [13] using different notation. Here we have repeated the proofs for completeness.

\section{A. Proof of Lemma 1}

First note that $\tilde{L} \leq L$ implies $X_{\tilde{L}} \subseteq X_{L}$. Thus, $\max _{\mathbf{x} \in X_{L}}\left\{\delta_{\mathbf{x}}^{\mathbf{u}}\right\} \geq \max _{\mathbf{x} \in X_{\tilde{L}}}\left\{\delta_{\mathbf{x}}^{\mathbf{u}}\right\}$. To complete the proof, we thus only need to show that $\max _{\mathbf{x} \in X_{L}}\left\{\delta_{\mathbf{x}}^{\mathbf{u}}\right\} \leq \max _{\mathbf{x} \in X_{\tilde{L}}}\left\{\delta_{\mathbf{x}}^{\mathbf{u}}\right\}$. To prove this, we show that for any $\mathbf{x} \in X_{L}$ there exists corresponding $\mathbf{y} \in X_{\tilde{L}}$ such that $V_{\mathbf{y}}^{*}-V_{\mathbf{y}}^{\mathbf{u}} \geq V_{\mathbf{x}}^{*}-V_{\mathbf{x}}^{\mathbf{u}}$. Consider any $\mathbf{x} \in X_{L}$. Note from the equation for $V_{\mathbf{x}}^{*}$ that for any realization $\mathbf{x}(\omega)$, where $\mathbf{x}=\left[x_{1}(\omega), \cdots, x_{N}(\omega)\right]$, only the $\min \left\{K_{0}, \sum_{l=1}^{N} I_{\left\{x_{l}(\omega)>0\right\}}\right\}$ available channels 
with highest rate determine the expected reward of the genie. We can define a new channel state $\mathbf{y}$ such that $y_{j}(\omega)=x_{j}(\omega)$ if and only if $x_{j}(\omega)>0$ and $j$ is one of the $\min \left\{K_{0}, \sum_{l=1}^{N} I_{\left\{x_{l}(\omega)>0\right\}}\right\}$ available channels with the highest rate. Otherwise, $y_{j}(\omega)=0$. We see that because $\sum_{l=1}^{N} I_{\left\{y_{l}(\omega)>0\right\}} \leq \tilde{L}$ for all realizations $\omega$, then $\mathbf{y} \in X_{\tilde{L}}$ and $V_{\mathbf{x}}^{*}=V_{\mathbf{y}}^{*}$. At the same time, $V_{\mathbf{x}}^{\mathbf{u}} \geq V_{\mathbf{y}}^{\mathbf{u}}$ for any strategy u. Thus, $V_{\mathbf{x}}^{*}-V_{\mathbf{x}}^{\mathbf{u}} \leq V_{\mathbf{y}}^{*}-V_{\mathbf{y}}^{\mathbf{u}}$.

\section{B. Proof of Lemma 2}

We use proof by contradiction. Suppose there exists $\mathbf{z} \in X_{\tilde{L}}$ such that $P\left(0<z_{j}<r_{j}\right)>0$ for some $j$ and $\delta^{\mathbf{u}}=V_{\mathbf{z}}^{*}-V_{\mathbf{z}}^{\mathbf{u}}$. Then from Lemma 1 we have the following: $\delta^{\mathbf{u}}=\max _{\mathbf{x} \in X_{\tilde{L}}}\left\{E_{\mathbf{u}} E_{\mathbf{x}}\left[\sum_{j=1}^{N} x_{j} I_{\left\{u_{j}=0\right\}}\right]\right\}=$ $\max _{\mathbf{x} \in X_{\tilde{L}}}\left\{\sum_{l=1}^{N} E\left[x_{l}\right] \cdot p_{u_{l}}(0)\right\}$. Now consider a modified channel state $\bar{z}$ such that for all $l, P\left(\bar{z}_{l}=r_{l}\right)=P\left(0<z_{l} \leq\right.$ $\left.r_{l}\right)$ and $P\left(\bar{z}_{l}=0\right)=P\left(z_{l}=0\right)$. Since $P\left(\bar{z}_{l} \geq z_{l}\right)=1$ for all $l$ and $P\left(\bar{z}_{j}>z_{j}\right)=1$ due to the assumption on $j$, we have: $\sum_{l=1}^{N} E\left[\bar{z}_{l}\right] \cdot p_{u_{l}}(0)>\sum_{l=1}^{N} E\left[z_{l}\right] \cdot p_{u_{l}}(0)$, which contradicts the assumption that the worst-case regret for $\mathbf{u}$ is achieved by $\mathbf{z}$. Since this result holds for any $\mathbf{z}$ such that $p\left(0<z_{j}<r_{j}\right)>0$ for some $j$, and clearly $\overline{\mathbf{z}} \in \bar{X}_{\tilde{L}}$, we have proven the result.

\section{Proof of Lemma 3}

Note that since Algorithm 1 randomly chooses which channels to use, then the value for $K$ after each step is a random variable. Let $K_{i}$ denote the value for $K$ after $i$ iterations of Step 1 . The value of $\bar{M}$ also changes after each iteration. We let $K_{0}$ and $M_{0}$ denote the initial (fixed) values for $K$ and $\bar{M}$, respectively. Thus we need to show that any strategy generated by Algorithm 1 has the same marginal probabilities as $\mathbf{u}\left[0, M_{0}\right]$ of Definition 1 , with $K_{0}$ replacing $K$. We first show this holds for $1 \leq i \leq M_{0}$ by induction.

Induction Basis: As described in Algorithm 1, initially $\tilde{p}=1-\frac{M_{0}-K_{0}}{r_{1} \sum_{j=1}^{M_{0}} \frac{1}{r_{j}}}$ and we set $u_{1}=1$ with probability $\tilde{p}$. Therefore, the result holds for $i=1$.

Induction Hypothesis: First consider any $2 \leq l \leq M_{0}$. Suppose the result holds for $i=1, \cdots, l-1$. We will show it also holds for $i=l$. The probability that we use channel $l$ is a random variable given by: $\tilde{p}=1-$ $\left[(\bar{M}-i+1)-K_{i-1}\right] /\left(r_{i} \sum_{j=i}^{M_{0}} \frac{1}{r_{j}}\right)$. Taking the expectation of $\tilde{p}$ gives the marginal probability that channel $i$ is used: $p_{u_{i}}(1)=1-\frac{\left(M_{0}-i+1\right)-E\left[K_{i-1}\right]}{r_{i} \sum_{j=i}^{M_{0}} \frac{1}{r_{j}}}$. We note that $E\left[K_{i-1}\right]$ can be calculated as follows by considering Step 2 of Algorithm 1: $E\left[K_{i-1}\right]=K_{0}-\sum_{j=1}^{i-1} p_{u_{j}}(1)=K_{0}-$ $\sum_{j=1}^{i-1}\left[1-\frac{M_{0}-K_{0}}{r_{j} \sum_{l=1}^{M_{0}} \frac{1}{r_{l}}}\right]$, where we have used the induction hypothesis to derive $p_{u_{j}}(1)$ for all $j \leq i-1$. Plugging this into the equation for $p_{u_{i}}(1)$ and rearranging, we obtain: $p_{u_{i}}(1)=1-\left[M_{0}-K_{0}\right] /\left[r_{i} \sum_{j=1}^{M_{0}} \frac{1}{r_{j}}\right]$, proving the result.

Now consider any $M_{0}+1 \leq l \leq N$. We need to prove that $p_{u_{l}}(1)=0$. By step 3 of the algorithm, this will always be true if $P\left(K_{M_{0}}=0\right)=1$. We prove this as follows. Initially,
$K_{0} \leq M_{0}$, and whenever $K_{j-1}=M_{0}-j+1$ then from Step 1 we have $\tilde{p}=1$ which means that in Step 2 we will obtain $K_{j}=K_{j-1}-1=M_{0}-(j+1)+1=M_{0}-j$. It is thus impossible to have $K_{j}>M_{0}-j$ for any $j$, implying $K_{M_{0}} \leq M_{0}-M_{0}=0$ with probability 1 .

\section{Proof of Theorem 2}

Consider any strategy $\mathbf{u}^{*}$ with marginal probabilities described in Theorem 2. It can be shown, similar to Lemma 1 , that the worst-case ratio of any strategy $\mathbf{u}$ is achieved by $\mathbf{x} \in X_{\tilde{L}}$ because the genie can only use the $\tilde{L}$ available channels with highest rates for transmission. Thus we have: $\rho^{\mathbf{u}^{*}}=\min _{\mathbf{x} \in X_{\tilde{L}}}\left\{\frac{K}{N} \sum_{j=1}^{N} E\left[x_{j}\right] / \sum_{j=1}^{N} E\left[x_{j}\right]\right\}=$ $\min _{\mathbf{x} \in X_{\tilde{L}}}\left\{\frac{K}{N}\right\}=K / N$. Now consider any other strategy $\mathbf{u}$ which does not probe channels uniformly. This implies there exists $1 \leq j \leq N$ such that $p_{u_{j}}(1) \neq K / N$. On the other hand, we know from definition of $U_{K}$ that $\sum_{j=1}^{N} p_{u_{j}}(1) \leq$ $K$. Thus, there exists $1 \leq i \leq N$ such that $p_{u_{i}}(1)<\frac{K}{N}$. Then we obtain for this $i$, by setting $p_{x_{i}}\left(r_{i}\right)=1$ and $p_{x_{j}}(0)=1$ for all $j \neq i: \rho^{\mathbf{u}}=\min _{\mathbf{x} \in \bar{X}_{L}}\left\{\frac{\sum_{j=1}^{N} E\left[x_{j}\right] p_{u_{j}}(1)}{\sum_{j=1}^{N} E\left[x_{j}\right]}\right\} \leq \frac{r_{i} p_{u_{i}}(1)}{r_{i}}<$ $\frac{K}{N}$. Thus we see that $\rho^{\mathbf{u}^{*}} \geq \rho^{\mathbf{u}}$ for all $\mathbf{u} \in U_{K}$, and therefore $\mathbf{u}^{*}$ must be optimal.

\section{E. Proof of Lemma 4}

First consider any strategy $\mathbf{u}[0, \bar{M}]$. From (4), $\delta^{\mathbf{u}}$ is given by the maximum of the following term over all $\mathbf{x} \in \bar{X}_{\tilde{L}}:\left\{\frac{(\bar{M}-K)}{\sum_{j=1}^{M} \frac{1}{r_{j}}} \sum_{i=1}^{\bar{M}} p_{x_{i}}\left(r_{i}\right)+\sum_{i=\bar{M}+1}^{N} r_{i} \cdot p_{x_{i}}\left(r_{i}\right)\right\}$. Meanwhile, by definition of $\eta(\bar{M})$, we have for all $1 \leq$ $i \leq \eta(\bar{M})<k$, where $i, k$ are integers: $r_{\bar{M}+i} \sum_{j=1}^{\bar{M}} \frac{1}{r_{j}} \geq$ $\bar{M}-K>r_{\bar{M}+k} \sum_{j=1}^{\bar{M}} \frac{1}{r_{j}}$. From these inequalities, there exists state $\mathbf{x}$ which achieves the maximum in $\delta^{\mathbf{u}}$ by setting $p_{x_{\bar{M}+j}}\left(r_{\bar{M}+j}\right)=0$ for $j \geq \eta(\bar{M})+1$, and $p_{x_{\bar{M}+j}}\left(r_{\bar{M}+j}\right)=$ 1 for $1 \leq j \leq \eta(\bar{M})$, and $p_{x_{j}}\left(r_{j}\right)=1$ for any $\tilde{L}-\eta(\bar{M})$ values of $j$ in $1 \leq j \leq \bar{M}$. Note this is a valid $\mathrm{x}$ as $\eta(\bar{M}) \leq \tilde{L}$ by definition of $\eta(\bar{M})$. Thus $\delta^{\mathbf{u}}$ reduces to the equation given in Lemma 4.

Consider $\mathbf{u}[l, \bar{M}]$ when $l>0$. In this case $\delta^{\mathbf{u}}$ can be calculated by using the following equation: $\delta^{\mathbf{u}}=$ $\max _{\mathbf{x} \in \bar{X}_{\tilde{L}}}\left\{\sum_{i=1}^{\bar{M}-1} r_{i} p_{x_{i}}\left(r_{i}\right) \frac{r_{\bar{M}+l}}{r_{i}}+\sum_{i=\bar{M}+1}^{N} r_{i} p_{x_{i}}\left(r_{i}\right)\right.$ $\left.+r_{\bar{M}} p_{x_{\bar{M}}}\left(r_{\bar{M}}\right)\left(\bar{M}-K-r_{\bar{M}+l} \sum_{j=1}^{\bar{M}-1} \frac{1}{r_{j}}\right)\right\}$. By the definition of $\eta(\bar{M}-1)$, we have the following: $r_{\bar{M}+\eta(\bar{M}-1)+1} \sum_{j=1}^{M-1} \frac{1}{r_{j}}<\bar{M}-1-K$. Combining this with $l \geq \eta(\bar{M}-1)$ and using $r_{j} \leq r_{k}$ for any $j \geq k$, we obtain the inequality: $r_{\bar{M}-1+l+1}\left(\sum_{j=1}^{\bar{M}} \frac{1}{r_{j}}\right)<(\bar{M}-K)$. Rearranging yields: $r_{\bar{M}+l}<r_{\bar{M}}\left(\bar{M}-K-\sum_{j=1}^{\bar{M}-1}\left(r_{\bar{M}+l} / r_{j}\right)\right)$.

Thus we see that $\delta^{\mathbf{u}}$ can be maximized by setting $p_{x_{\bar{M}+k}}\left(r_{\bar{M}+k}\right)=1$ for $0 \leq k \leq l-1$, and $p_{x_{j}}\left(r_{j}\right)=1$ for any $\tilde{L}-l$ values of $j$ in $1 \leq j \leq \bar{M}-1$. Plugging this maximization into the previously stated equation for $\delta^{\mathbf{u}}$ completes the proof. 


\section{F. Optimal Strategy for Problem 1}

We note from the definition of $M^{*}$ from case 1 of Theorem 1 that $0 \leq p_{x_{j}}\left(r_{j}\right) \leq 1$, i.e. these are valid probabilities. Since $\min _{\mathbf{u} \in U_{K}}\left\{V_{\mathbf{x}}^{*}-V_{\mathbf{x}}^{\mathbf{u}}\right\}=V_{\mathbf{x}}^{*}-\max _{\mathbf{u} \in U_{K}} V_{\mathbf{x}}^{\mathbf{u}}$, the optimal strategy for this problem maximizes $V_{\mathbf{x}}^{\mathbf{u}}$. Plugging the marginal probabilities for $\mathbf{x}$, we obtain $V_{\mathbf{x}}^{\mathbf{u}}=$ $\sum_{j=1}^{M^{*}} p_{u_{j}}(1) \frac{\tilde{L}-\eta\left(M^{*}\right)}{\sum_{j=1}^{M} \frac{1}{r_{j}}}+\sum_{j=1}^{\eta\left(M^{*}\right)} p_{u_{j}}(1) r_{M^{*}+j}$. We see from the definition of $M^{*}$ in case 1 of Theorem 1 that there exists a strategy which maximizes $V_{\mathbf{x}}^{\mathbf{u}}$ by setting $p_{u_{j}}(1)=0$ for all $M+1 \leq j \leq N$ and probes any $K$ of the channels in $\left\{1, \cdots, M^{*}\right\}$. Thus, the reward of optimal strategy $\mathbf{u}^{*}$ is given by: $V_{\mathbf{x}}^{\mathbf{u}^{*}}=\left[\tilde{L}-\eta\left(M^{*}\right)\right] K /\left(\sum_{j=1}^{M^{*}} \frac{1}{r_{j}}\right)$, which holds regardless of which $K$ channels are probed. Meanwhile, $V_{\mathbf{x}}^{*}=\sum_{l=1}^{N} r_{l} p_{x_{l}}\left(r_{l}\right)=\frac{\tilde{L}-\eta\left(M^{*}\right)}{\sum_{j=1}^{M^{*}} \frac{1}{r_{j}}} M^{*}$. Taking the difference between $V_{\mathbf{x}}^{*}$ and $V_{\mathbf{x}}^{\mathbf{u}^{*}}$, and comparing with $\delta^{\mathbf{u}}\left[0, M^{*}\right]$ given by Lemma 4 , we obtain the result.

\section{G. Optimal Strategy for Problem 2}

We follow a similar technique used in Appendix $\mathrm{F}$ for Problem 1. First, $V_{\mathbf{x}}^{\mathbf{u}}$ can be written as: $V_{\mathbf{x}}^{\mathbf{u}}=\sum_{j=1}^{M^{*}} \frac{r_{M^{*}}}{r_{j}} p_{u_{j}}(1) r_{j}+\sum_{j=1}^{l-1} p_{u_{M+j}}(1) r_{M+j} \quad+$ $\left(\tilde{L}-l-r_{M^{*}} \sum_{j=1}^{M^{*}-1} \frac{1}{r_{j}}\right) p_{u_{M^{*}+l}}(1) r_{M^{*}+l}, \quad$ and the optimal strategy maximizes this. We see that because $r_{j}>r_{k}$ for all $j<k$, then $V_{\mathbf{x}}^{\mathbf{u}}$ is maximized by probing any $K$ of the channels in $\left\{0, \cdots, M^{*}\right\}$. Thus its reward is simply $r_{M^{*}} K$. Meanwhile, $V_{\mathbf{x}}^{*}=$ $r_{M^{*}} M^{*}+\sum_{j=1}^{l-1} r_{M+j}+\left[\tilde{L}-l-r_{M^{*}} \sum_{j=1}^{M^{*}-1} \frac{1}{r_{j}}\right] r_{M^{*}+l}$. Taking the difference between this and $r_{M^{*}} K$, then comparing with $\delta^{\mathbf{u}\left[l, M^{*}\right]}$ in Lemma 4 , proves the result.

\section{H. Proof of Theorem 3}

The proof of Theorem 3 is similar to the proof of Theorem 1 provided in Section IV-C. Therefore, only a sketch proof is provided in this section. It can be shown similarly to Lemma 1 that $\left\{V_{\mathbf{y}}^{*}-V_{\mathbf{y}}^{\mathbf{u}}\right\}$ is maximized for some $\mathbf{y} \in Y_{\tilde{L}}$. Additionally, it can be shown that because $r_{1}$ satisfies the inequality described by the theorem, and from the definition of $M$ and $\eta(\cdot)$, we have $q \cdot r_{1} \leq$ $p \cdot r_{M^{*}+\eta\left(M^{*}\right)}$. Thus for any strategy which only probes channels in $\left\{1,2, \cdots, M^{*}+\eta\left(M^{*}\right)\right\}$, we have $\max _{\mathbf{y} \in Y_{\tilde{L}}}\left\{V_{\mathbf{y}}^{*}-\right.$ $V_{\mathbf{y}}^{\mathbf{u}}=\max _{\mathbf{y} \in Y_{\tilde{L}}}\left\{\sum_{j=1}^{N} p_{y_{j}}(1) r_{j}\left[p_{u_{j}}(0)(p-q)+q\right]\right\}-$ $\sum_{j=1}^{N} p_{u_{j}}(1) r_{j} q$.
It can be shown, using steps similar to the proof of Lemma 3, that for any $\mathbf{u}$ in Theorem 3, we have the following: $\max _{\mathbf{y} \in Y_{\tilde{L}}}\left\{V_{\mathbf{y}}^{*}-V_{\mathbf{y}}^{\mathbf{u}}\right\}=\frac{\left[\left(\tilde{L}-\eta\left(M^{*}\right)\right)(p-q)+M^{*} q\right]}{(p-q) \sum_{k=1}^{M^{*}} \frac{1}{r_{k}}}\left[\left(M^{*}-\right.\right.$ $K) p+q K]+p \sum_{j=1}^{\eta\left(M^{*}\right)}\left[r_{M^{*}+j}-\frac{q}{p-q} r_{j}\right]$. Now consider the following:

Problem 3: For any $\tilde{L}, K$, suppose the conditions of Theorem 3 are satisfied for some $M^{*}$. For $1 \leq j \leq M^{*}$, let $p_{y_{j}}(1)=\frac{\left[\tilde{L}-\eta\left(M^{*}\right)\right] p+\left(M^{*}-\tilde{L}+\eta\left(M^{*}\right)\right) q}{r_{j}(p-q) \sum_{k=1}^{M^{*}} \frac{1}{r_{k}}}-\frac{q}{p-q}$. For $M^{*}+1 \leq$ $j \leq M^{*}+\eta\left(M^{*}\right)$, let $p_{y_{j}}(1)^{k}=1$. Finally, let $p_{y_{j}}(1)=0$ for $j>M^{*}+\eta\left(M^{*}\right)$.

It can be shown the optimal strategy probes any $K$ channels in $\left\{1, \cdots, M^{*}\right\}$, and $\min _{\mathbf{u} \in U_{K}}\left\{V_{\mathbf{y}}^{*}-V_{\mathbf{y}}^{\mathbf{u}}\right\}$ matches $\max _{\mathbf{y} \in Y_{\tilde{L}}}\left\{V_{\mathbf{y}}^{*}-V_{\mathbf{y}}^{\mathbf{u}}\right\}$ calculated earlier. Combining this with (6) proves Theorem 3.

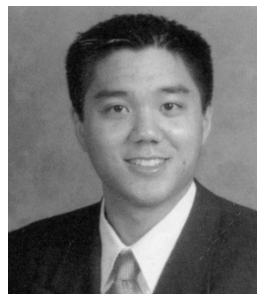

Nicholas B. Chang received the B.S.E. degree in electrical engineering (magna cum laude) from Princeton University, Princeton, NJ in 2002, and the M.S.E degree in electrical engineering: systems, M.S. degree in mathematics, and Ph.D. degree in electrical engineering: systems, from the University of Michigan, Ann Arbor, in 2004, 2005, and 2007 respectively. He is currently a technical staff member at MIT Lincoln Laboratory in Lexington, MA. His research interests include communication networks, wireless communication, stochastic control, stochastic resource allocation, and algorithms. Dr. Chang is a Member of Tau Beta Pi and Sigma Xi, the Scientific Research Society. He is a recipient of the 2005-2006 MIT Lincoln Laboratory Graduate Fellowship.

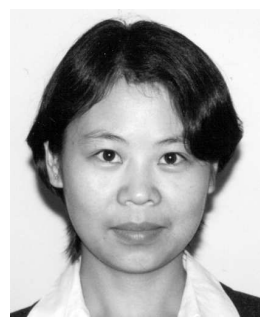

Mingyan Liu (M'00 / ACM'01) received her B.Sc. degree in electrical engineering in 1995 from the Nanjing University of Aero. and Astro., Nanjing, China, M.Sc. degree in systems engineering and Ph.D. Degree in electrical engineering from the University of Maryland, College Park, in 1997 and 2000, respectively. She joined the Department of Electrical Engineering and Computer Science at the University of Michigan, Ann Arbor, in September 2000, where she is currently an Associate Professor. Her research interests are in performance modeling, analysis, energy-efficiency and resource allocation issues in wireless, mobile ad hoc, and sensor networks. She is the recipient of the 2002 NSF CAREER Award, and the University of Michigan Elizabeth C. Crosby Research Award in 2003. She serves on the editorial board of IEEE/ACM Transactions on Networking and IEEE Transactions on Mobile Computing. 